Pacific Journal of Mathematics

ENUMERATING PSEUDO-ANOSOV FOLIATIONS 


\title{
ENUMERATING PSEUDO-ANOSOV FOLIATIONS
}

\author{
Athanase Papadopoulos and Robert C. Penner
}

\begin{abstract}
Let $M$ be a closed oriented surface of genus at least two, and let $\mathscr{P} \mathscr{F}$ denote the space of all projective classes of measured foliations on $M$. The authors have previously given a criterion in terms of certain combinatorial words for an element of $\mathscr{P} \mathscr{F}$ to be left invariant by a pseudo-Anosov map of $M$ : such foliations are characterized by the fact that the associated word is eventually periodic. The current work derives an estimate which says roughly that the dilatation of the corresponding pseudo-Anosov map is large if the periodic part of the word is long. This estimate is then used to bound the number of distinct conjugacy classes of foliations invariant under pseudo-Anosov maps of $M$ in terms of a specified bound on the dilatations.
\end{abstract}

1. Introduction. This paper is a sequel to our paper [5] in which we described a method of representing measured foliations carried by a train track by means of semi-infinite combinatorial words in a finite alphabet. We showed that pseudo-Anosov foliations (i.e., those that are preserved by pseudo-Anosov maps) are characterized by being representable by eventually periodic "convergent" words. In this paper, we establish an inequality relating the length of the repeating part of the word corresponding to a pseudo-Anosov foliation and the dilatation factor of a pseudo-Anosov map preserving that foliation. As a by-product, for each real number $R$, we shall describe a finite set of foliations, whose cardinality is bounded in terms of $R$ and which contains a representative of each conjugacy class of pseudo-Anosov foliation which is preserved by a pseudo-Anosov map of dilatation at most $R$.

Throughout this paper, $M$ will denote a closed, oriented, smooth surface of negative Euler characteristic. Moreover, unless otherwise stated, we will tacitly assume that each train track considered is trivalent, i.e., there are exactly three half-branches incident on each switch. For the basic facts about measured foliations and train tracks, we refer the reader to [1], [3], [4], [5], or [6]. It is necessary to further refine some of the notions developed in [5] (whose terminology we will adopt), and this is the purpose of the next paragraph. 
2. Directed RLS Words. Given a train track $\tau \subset M$, we shall employ three elementary moves, each of which produces from $\tau$ another train track $\tau^{\prime}$ carried by $\tau$. These moves are called right splits, left splits, and shifts and are indicated in Figure 1a-c, respectively; in each case, the elementary move is said to be performed along the branch labeled $e$.

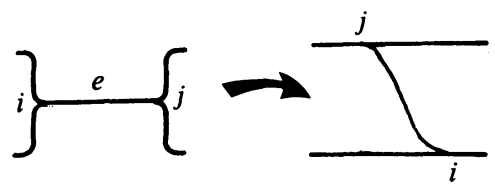

(a)

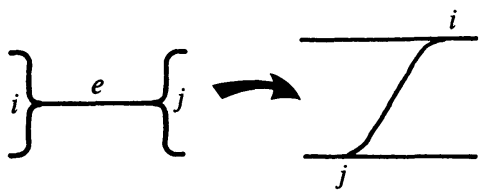

(b)

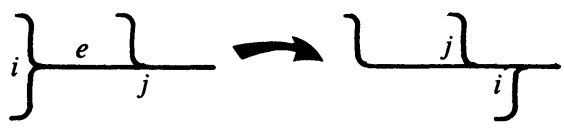

(c)

FIGURE 1

Suppose that $\tau$ has $I$ switches. A labeling of $\tau$ is a bijection from the set of switches of $\tau$ to the set $\{1, \ldots, I\}$. Notice that if $\tau^{\prime}$ is a train track obtained from $\tau$ by an elementary move, then there is a natural one-to-one correspondence between the set of switches of $\tau$ and those of $\tau^{\prime}$, so a labeling of $\tau$ induces a labeling of $\tau^{\prime}$, as indicated in Figure 1. Fix a labeling of $\tau$ once and for all, and consider the finite alphabet

$$
\mathscr{A}_{I}=\left\{R_{1}, L_{1}, S_{1}, \ldots, R_{I}, L_{I}, S_{I}\right\} .
$$

Suppose that

$$
\tau=\tau_{0}, \tau_{1}, \ldots, \tau_{K}
$$

is a finite sequence of train tracks, where $\tau_{k+1}$ arises from $\tau_{k}$ by an elementary move, for $k=0, \ldots, K-1$. This sequence can be codified by a finite word $w$ in the alphabet $\mathscr{A}_{I}$ as follows. The first letter of $w$ is given by $X_{i}$, where $X=R, X=L$, or $X=S$ if the elementary move applied to $\tau_{0}$ is a right split, a left split, or a shift, respectively, along the branch $e$. The subscript $i \in\{1, \ldots, I\}$ is chosen in such a way that the branch $e$ is incident on the $i$ th switch (relative to the labeling). In the case of a shift, we furthermore require that any smooth arc in $\tau$ which meets the interior of $e$ must pass through the $i$ th switch; for instance, the shift in Figure 1c gives rise to the letter $S_{i}$, not $S_{j}$. Thus, the subscript $i$ is uniquely determined in the case of a shift, and there are two possible choices of subscript in the case of a right or a left 
split. Of course, $\tau_{1}$ inherits a labeling from that of $\tau_{0}$, as above. In general, the $k$ th letter of $w$, for $K-1 \geq k \geq 2$, is determined in an analogous manner relative to the induced labeling on $\tau_{k-1}$, and the definition of $w$ is complete. If a finite word $w$ in the alphabet $\mathscr{A}_{I}$ arises as above from some consecutive sequence of elementary moves applied to $\tau$, then we say that $w$ is a (finite) $R L S$ word on $\tau$.

Notice that not all finite words in the alphabet $\mathscr{A}_{I}$ arise as RLS words on $\tau$ (since shifts and splits can be performed only along certain types of branches), and, if a consecutive sequence of elementary moves applied to $\tau$ involves $\Sigma$ splits, then there are $2^{\Sigma}$ associated RLS words on $\tau$ (owing to our indeterminacy in choice of subscript in case of splits). On the other hand, a finite RLS word $w$ on $\tau$ consisting of $K-1$ letters uniquely determines the associated sequence $\tau=\tau_{0}, \ldots, \tau_{K}$ of train tracks, and there is a well-defined train track $\tau(w)=\tau_{K}$ carried by $\tau$ which is associated to the RLS word $w$. A finite RLS word $w$ on $\tau$ is said to be directed if, whenever two letters of $w$ have the same subscript, then all the letters of $w$ occurring between these two letters also have that same subscript.

Let $N(\tau)$ be a regular neighborhood of $\tau$ in $M$ equipped with its foliation $\mathscr{T}$ by ties. A leaf of $\mathscr{T}$ is said to be singular if it passes through a cusp of $\partial N(\tau)$; see Figure 2 .

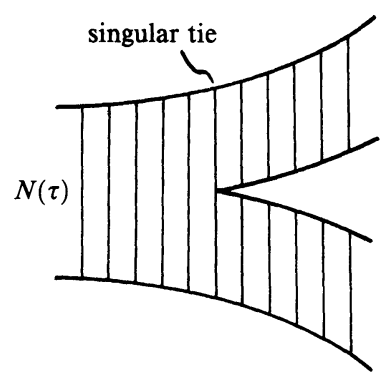

FIGURe 2

Let $I$ denote the number of switches of $\tau$. We say that $N(\tau)$ is suited to a train track $\tau^{*}$ provided that $\tau^{*}$ is contained in the interior of $N(\tau)$ transverse to the ties, no switch of $\tau^{*}$ lies on a singular tie of $N(\tau)$, no two distinct switches of $\tau^{*}$ lie on a common tie of $\mathscr{T}$, and there is a collection of arcs $\left\{\Gamma_{1}, \ldots, \Gamma_{I}\right\}$ disjointly imbedded in $N(\tau)$ so that the following conditions hold.

(1) The interior of each arc in the collection is disjoint from $\tau^{*}$.

(2) Each arc $\Gamma$ in the collection has one endpoint at some cusp $c$ of $\partial N(\tau)$ and the other endpoint at some switch $s$ of $\tau^{*}$. Furthermore, in 
a neighborhood of $c, \Gamma$ and $\partial N(\tau)$ lie on opposite sides of the singular tie through $c$, and, in a neighborhood of $s, \Gamma$ approaches $s$ between the branches of $\tau^{*}$ which meet with zero angle at $s$. See Figure 3.

(3) For each switch $s$ of $\tau^{*}$, there is a (necessarily unique) arc in the collection with $s$ as endpoint.

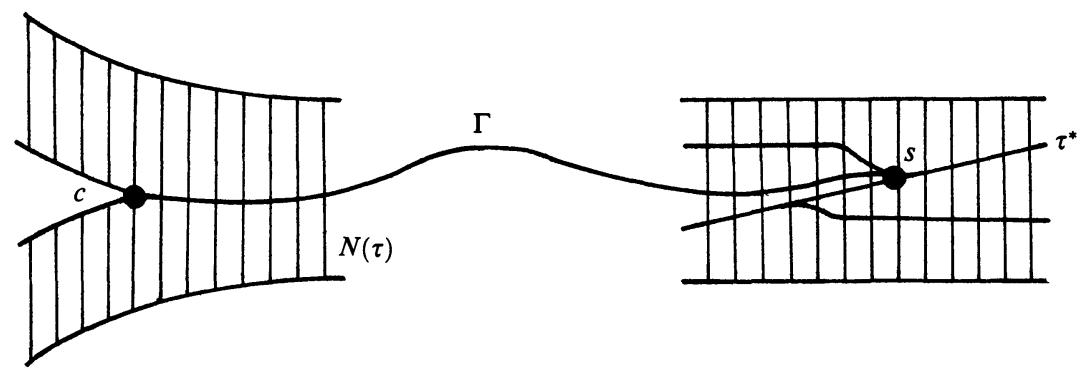

FIGURE 3

Such a collection $\left\{\Gamma_{1}, \ldots, \Gamma_{I}\right\}$ is called a pairing arc system for $\tau$ and $\tau^{*}$, and each element of a pairing arc system is called a pairing arc. Notice that a pairing arc system induces a bijection between the set of switches of $\tau$ and the set of switches of $\tau^{*}$.

We next recall the "unzipping construction" (from $\S 2.3$ of [5]), which associates a finite directed RLS word to $\tau^{*}$ when $N(\tau)$ is suited to $\tau^{*}$. In fact, there is a directed RLS word corresponding to each linear ordering of the set of arcs in the pairing arc system $\left\{\Gamma_{1}, \ldots, \Gamma_{I}\right\}$, and we describe the directed RLS word associated to the order $\Gamma_{1}, \ldots$, $\Gamma_{I}$. Let us parametrize each $\Gamma_{i}$ by a map

$$
\gamma_{i}:[i-1, i] \rightarrow \Gamma_{i}
$$

so that $\gamma_{i}(i-1)$ is a cusp of $\partial N(\tau)$ and $\gamma_{i}(i)$ is a switch of $\tau^{*}$, and let $\hat{\gamma}_{i}$ denote the restriction of $\gamma_{i}$ to the half-open interval [ $i-1, i[$, for $i=1, \ldots, I$. For each $t \geq 0$, define

$$
N_{t}=N(\tau)-\bigcup_{i=1}^{[t]+1}\left\{\hat{\gamma}_{i}(s): s<t\right\}
$$

where $[t]$ denotes the integral part of $t$, and notice that the foliation $\mathscr{T}$ of $N(\tau)$ by ties restricts to a foliation $\mathscr{T}_{t}$ of $N_{t}$, for each $t \geq 0$. 
Since the pairing arcs are pairwise disjoint, there is an associated collection $\Gamma_{i}^{t}=\Gamma_{i} \cap N_{t}$, for $i=1, \ldots, I$ of (oriented) arcs disjointly imbedded in $N_{t}$, for each $0 \leq t \leq I$. The singular ties of $N_{t}$ are those leaves of $\mathscr{T}_{t}$ which contain the initial point of $\Gamma_{i}^{t}$, for some $i=$ $1, \ldots, I$, and a parameter $t$ is called a critical time if some singular tie of $N_{t}$ contains the initial points of two distinct arcs $\left\{\Gamma_{i}^{t}\right\}_{i=1}^{I}$. We may collapse each leaf of $\mathscr{T}_{t}$ to a point to produce from $N_{t}$ a train track $\tau_{t}$ (so that singular ties of $\mathscr{T}_{t}$ give rise to switches of $\tau_{t}$ ); see Figure 4. Notice that $\tau_{t}$ is trivalent precisely when the parameter $t$ is not a critical time.
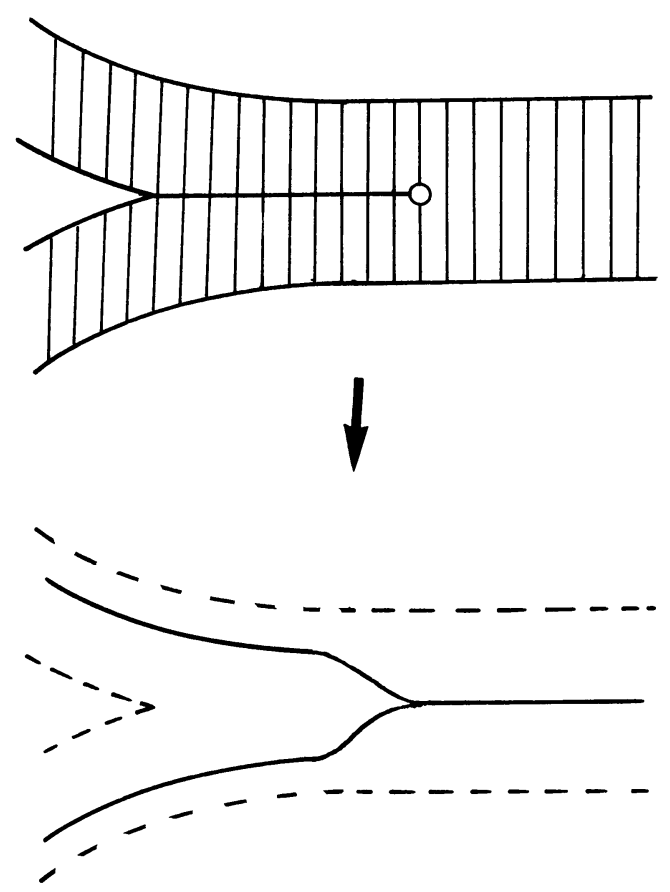

FIGURE 4

It is clear that if $t$ is not a critical time, then $\tau_{s}$ is isotopic to $\tau_{t}$ provided that $s$ is sufficiently close to $t$. On the other hand, if $t$ is a critical time and $\varepsilon>0$ is sufficiently small, then the train track $\tau_{t+\varepsilon}$ arises from the train track $\tau_{t-\varepsilon}$ by an elementary move; see Figures $5 \mathrm{a}$ and $\mathrm{b}$ for the cases of shifts and splits, respectively. Notice that a singular tie of $N_{t+\varepsilon}$ either contains the initial point of $\Gamma_{[t]+1}^{t}$ or is contained in a singular tie of $N_{[t]}$, as indicated in Figure 5. 

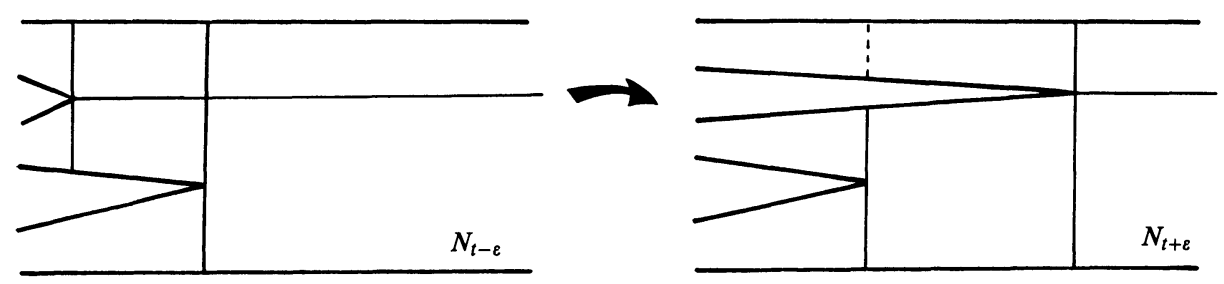

(a)
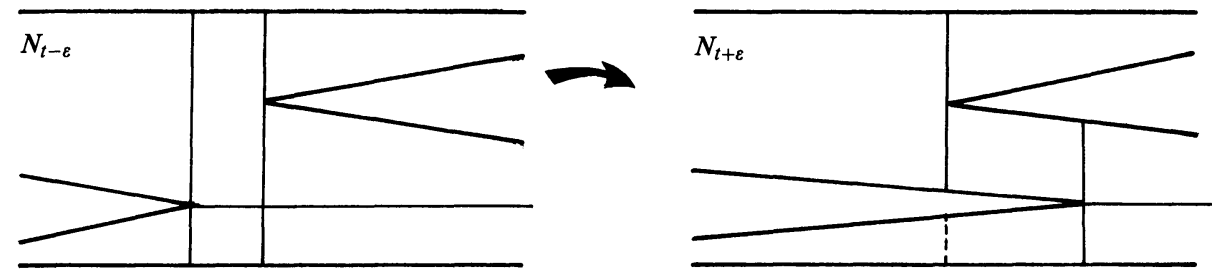

(b)

FIGURE 5

It follows that if $t_{1}<\cdots<t_{K}$ denote the critical times and $\varepsilon>0$ is sufficiently small, then the sequence of train tracks

$$
\tau_{t_{1}-\varepsilon}, \tau_{t_{2}-\varepsilon}, \ldots, \tau_{t_{K}-\varepsilon}, \tau_{t_{K}+\varepsilon}
$$

arise from consecutive application of elementary moves. Since $\tau$ is isotopic to $\tau_{t_{1}-\varepsilon}$ and $\tau^{*}$ is isotopic to $\tau_{t_{K}+\varepsilon}$, there is a corresponding finite directed RLS word $w$ on $\tau$ so that $\tau^{*}=\tau(w)$, completing our discussion.

3. Equivalent RLS words. Two finite RLS words $w$ and $w^{\prime}$ on $\tau$ are said to be equivalent if the two train tracks $\tau(\omega)$ and $\tau\left(w^{\prime}\right)$ are isotopic.

Proposition 1. Every finite RLS word is equivalent to a directed finite RLS word.

Proof. This is a consequence of the discussion of the zipper construction in $\S 2$ and the fact that if $\tau^{\prime}$ is a train track obtained from $\tau$ by a finite sequence of elementary moves and $N(\tau)$ is a tie neighborhood of $\tau$, then $\tau^{\prime}$ may be isotoped to a train track to which $N(\tau)$ is suited.

Notice that we can uniquely determine a directed RLS word in each equivalence class of finite RLS words if we stipulate that the linear 
ordering on the pairing arc system is induced from the labeling on $\tau$, and, whenever each of $X_{i}$ and $X_{j}$ (where $X \in\{R, L\}$ ) are legitimate possibilities for a given letter of an RLS word, then we choose $X_{\inf \{i, j\}}$.

4. Arc-length and word-length. Fix a train track $\tau \subset M$ and a regular neighborhood $N(\tau)$ of $\tau$ foliated by ties. If $L$ is an arc imbedded in $N(\tau)$ transverse to the ties, then we define the $(\operatorname{arc})$ length $l_{N(\tau)}(L)$ of $L$ with respect to $N(\tau)$ to be the number of intersection points of $L$ with the union of the singular ties of $N(\tau)$. Analogously, if $w$ is a finite RLS word associated to $\tau$, then we define the (word) length $l(w)$ of $w$ to be the total number of letters comprising $w$.

Proposition 2. Suppose that the regular neighborhood $N(\tau)$ of the train track $\tau \subset M$ is suited to the train $\tau^{*}$, and let $\left\{\Gamma_{1}, \ldots, \Gamma_{I}\right\}$ be the corresponding pairing arc system. Let $w$ be the directed finite $R L S$ word associated to unzipping $N(\tau)$ successively along $\Gamma_{1}, \ldots, \Gamma_{I}$, in this order, and, for each $i=1, \ldots, I$, let $w_{i}$ denote the sub-string of $w$ which arises from unzipping along the arc $\Gamma_{i}$. For each $i=1, \ldots, I$, we have the inequality

$$
l\left(w_{i}\right) \leq 2^{i-1} l_{N(\tau)}\left(\Gamma_{i}\right)
$$

For the sequel, we shall only need the following estimate.

Corollary 3. In the situation described in Proposition 2 , there is a constant $C$ depending only on the Euler characteristic of the surface $M$ so that

$$
l(w) \leq C \sum_{i=1}^{I} l_{N(\tau)}\left(\Gamma_{i}\right)
$$

Proof of Corollary 3. The number of switches of a train track is bounded by an integer $M_{0}$ depending only on the Euler characteristic of the surface $M$; in fact, one can take $M_{0}=-6 \chi(M)$. The result then follows directly from Proposition 2 by taking $C=2^{M_{0}-1}$.

Proof of Proposition 2. Adopt the notation of $\S 2$ above for the train tracks $\tau_{t}$ arising from $N_{t} \subset N(\tau)$ and the arcs $\Gamma_{i}^{t}=\Gamma_{i} \cap N_{t}$, for $i=$ $1, \ldots, I$ and $t \geq 0$. Since the interior of each pairing arc is disjoint from $\tau^{*}$, the collection $\left\{\Gamma_{i}^{t}\right\}_{i=1}^{I}$ forms a pairing arc system for $\tau^{*}$ with respect to the neighborhood $N_{t}$ of $\tau_{t}$, and $N_{t}$ is suited to $\tau^{*}$. 
The assertion in the proposition follows easily from the following two estimates:

$$
\begin{aligned}
& l\left(w_{i}\right) \leq l_{N_{i-1}}\left(\Gamma_{i}\right), \quad \text { for } i=1, \ldots, I \\
& l_{N_{l}}\left(\Gamma_{j}\right) \leq 2 l_{N_{i-1}}\left(\Gamma_{j}\right), \quad \text { for } I \geq i \geq 1 .
\end{aligned}
$$

Since a singular tie of $N_{t}$ which does not contain the initial point of $\Gamma_{[t]+1}^{t}$ is contained in a singular tie of $N_{[t]}$, it follows that $l_{N_{i-1}}$ majorizes the number of critical times in the interval $[i-1, i]$. Insofar as this last quantity agrees with $l\left(w_{i}\right)$, the estimate $(*)$ follows.

For the estimate $(* *)$, consider $l_{N_{t}}\left(\Gamma_{j}\right)$ as a function of $t$ for $t \in$ $[i-1, i]$. If $t \in[i-1, i]$ is not a critical time and $l_{N_{t}}\left(\Gamma_{j}\right)$ is not continuous at $t$, then $\Gamma_{i}^{t}$ must contain the terminal point of $\Gamma_{j}$, and $l_{N_{t}}\left(\Gamma_{j}\right)$ has a jump discontinuity of magnitude +1 at $t$; see Figure 6a. If $t \in[i-1, i]$ is a critical time, then the terminal point of $\Gamma_{j}$ cannot lie on $\Gamma_{i}^{t}$ by our conditions on a pairing arc system. There are two basic cases depending on whether the elementary move associated to the critical time $t$ is a split or a shift; the various sub-cases for these possibilities, respectively, are illustrated in Figures $6 \mathrm{~b}$ and $\mathrm{c}$. In each case, either $l_{N_{t}}\left(\Gamma_{j}\right)$ is continuous at $t$ or perhaps has a jump discontinuity of magnitude +1 or -1 at $t$.

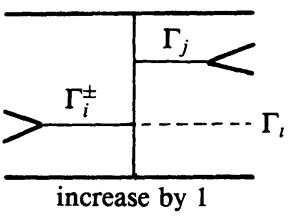

(a)

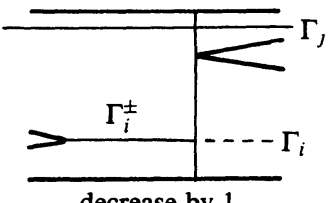

decrease by 1

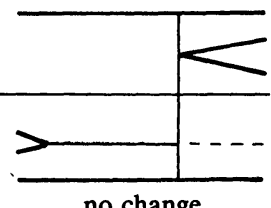

no change

(b)

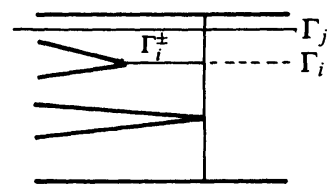

decrease by 1

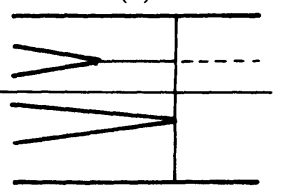

no change

(c)
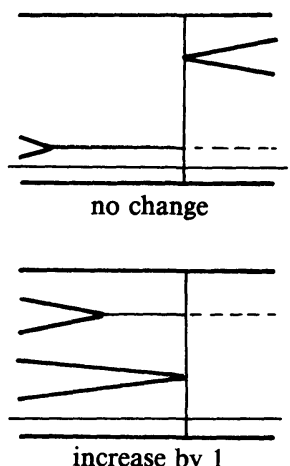

increase by 1

Figure 6 
Now, Let us write $l_{N_{t}}\left(\Gamma_{j}\right)=l(t)+l^{\prime}(t)$, where $l(t)$ denotes the number of times that $\Gamma_{j}$ meets the singular leaf of $N_{t}$ containing the endpoint of $\Gamma_{i}^{t}$, and $l^{\prime}(t)$ denotes the number of intersections of $\Gamma_{j}$ with the other singular leaves of $N_{t}$. Inspection of Figure 6 shows that $l^{\prime}(t)$ is non-increasing in $t$, so $l^{\prime}(i) \leq l^{\prime}(i-1) \leq l_{N_{l-1}}\left(\Gamma_{j}\right)$. On the other hand, for each non-critical time $t$, the singular leaf of $N_{t}$ containing the endpoint of $\Gamma_{i}^{t}$ lies in the interior of a rectangle in $N_{i-1}$, two of whose opposite sides are contained in singular leaves of $N_{i-1}$. It follows easily that $l^{\prime}(t)$ is also majorized by $l_{N_{l-1}}\left(\Gamma_{j}\right)$, so, in particular, $l^{\prime}(i) \leq l_{N_{t-1}}\left(\Gamma_{j}\right)$. Thus,

$$
l_{N_{l}}\left(\Gamma_{j}\right)=l(i)+l^{\prime}(i) \leq 2 l_{N_{i-1}}\left(\Gamma_{j}\right) \text {, }
$$

and the proof of estimate $(* *)$ and hence the proof of Proposition 2 is complete.

Proposition 4. Let $w$ be a finite directed $R L S$ word on a train track $\tau \subset M$. There is a finite directed $R L S$ word $w^{\prime}$ on $\tau$ which is equivalent to $w$ with the property that if $\Gamma$ is the first pairing arc in the unzipping corresponding to $w^{\prime}$, then

$$
l_{N(\tau)}(\Gamma) \geq l(w) / C_{1},
$$

where $C_{1}$ is a constant which depends only on the Euler characteristic of $M$.

Proof. It follows from Corollary 3 that

$$
\frac{l(w)}{C} \leq \sum_{i=1, \ldots, I} l_{N(\tau)}\left(\Gamma_{i}\right),
$$

so there is some $i \in\{1, \ldots, I\}$ so that

$$
l_{N(\tau)}\left(\Gamma_{i}\right) \geq \frac{1}{C I} l(w) .
$$

Of course, $I \leq M_{0}$, and we find that

$$
l_{N(\tau)}\left(\Gamma_{i}\right) \geq \frac{l(w)}{C_{1}}
$$

where $C_{1}=M_{0} 2^{M_{0}-1} \leq-6 \chi(M) 2^{6 \chi(M)-1}$. Finally, we may choose a new line ordering on the set of pairing arcs so that the pairing $\operatorname{arc} \Gamma_{i}$ is first, and take for $w^{\prime}$ the corresponding directed RLS word.

We shall also need the following estimate which gives a lower bound on word length in terms of arc length. 
Proposition 5. Under the hypotheses of Proposition 2, we have the estimate

$$
l\left(w_{1}\right) \geq\left[\frac{\log l_{N(\tau)}\left(\Gamma_{1}\right)}{\log 3}\right]
$$

where $[x]$ denotes the integral part of $x$.

Proof. Suppose that $t \in[0,1]$ is the unique critical time in the interval $\left[t_{-}, t_{+}\right]$, and define the quantities

$$
l_{+}=l_{N_{t_{+}}}\left(\Gamma_{1}^{t_{+}}\right) \text {and } l_{-}=l_{N_{-}}\left(\Gamma_{1}^{t_{-}}\right) .
$$

Provided that $l_{+}$exceeds one, there must be another critical time in the interval $\left[t_{+}, 1\right]$, and hence the unzipping of $N_{t_{+}}$along $\Gamma_{1}^{t_{+}}$will generate yet another letter of $w_{1}$.

We claim that

$$
l_{+} \geq \frac{l_{-}-1}{2}
$$

To see this, consider the singular ties of $N_{t_{-}}$and $N_{t_{+}}$, as illustrated in Figures $5 \mathrm{a}$ and $\mathrm{b}$ corresponding to the cases in which the elementary move associated to the critical time $t$ is a shift or a split, respectively. In either case, the relative complement of the set of all singular ties of $N_{t_{-}}$in the set of all singular ties of $N_{t_{+}}$consists of the singular tie containing the initial point of $\Gamma_{1}^{t_{+}}$together with a unique (closed) arc $\alpha$, which is indicated by a dotted line in Figure 5. Clearly, $l_{-}-l_{+}=1+q$, where $q$ denotes the number of intersection points of $\Gamma_{1}^{t_{-}}$with the interior of the arc $\alpha$ (and the summand 1 arises from the intersection of $\Gamma_{1}^{t_{-}}$with an endpoint of $\alpha$ ). Now, $\alpha$ is contained in a rectangle $R \subset N_{t_{-}}$which is bounded on two opposite sides by singular ties of $N_{t_{-}}$, and each component of $\Gamma_{1}^{t_{-}} \cap R$ either meets both these sides of $R$ or has one endpoint (namely, the terminal point of $\Gamma_{1}^{t_{-}}$) in the interior of $R$. There can be at most one component of the latter type, and it follows that $q \leq\left(l_{-}-1\right) / 2$. Thus, $l_{-}-l_{+}=1+q \leq\left(l_{-}+1\right) / 2$, proving the claim.

Finally, provided that $l_{-} \geq 3$, we have the inequality $\left(l_{-}-1\right) / 2 \geq$ $l_{-} / 3$, so

$$
l_{+} \geq l_{-} / 3
$$

and the result follows easily.

5. Word length and dilatation. Recall from [5] that one may associate a "semi-infinite RLS word" $w$ in the alphabet $\mathscr{A}_{i}$ to a suitable measured foliation $\mathscr{F}$ carried by a train track $\tau$ with $I$ switches, and the word $w$ is said to be "convergent" if it contains infinitely many 
letters of the type $R$ or $L$. By Theorem 3.1 and Corollary 4.3 of [5], if the word $w$ is periodic and convergent, then $w$ uniquely determines the equivalence class of $\mathscr{F}$. (This is not true if $w$ is not convergent.) In fact, if $w$ is periodic and convergent, then the measure on $\tau$ corresponding to $\mathscr{F}$ is an eigenvector of a certain "incidence matrix", (defined below) and the corresponding eigenvalue is equal to the spectral radius of this incidence matrix; we refer the reader to [5] for the details.

Our main application of the estimates in $\S 4$ follows.

THeOREM 6. Fix a surface $M$. Given $\lambda>1$, there is an integer $\Lambda$ depending only on $\lambda$ and the Euler characteristic $\chi(M)$ of $M$ so that the following condition holds: if $\mathscr{F}$ is a pseudo-Anosov foliation which is left invariant by a pseudo-Anosov map of $M$ of dilatation $\lambda$, then there is a train track $\tau \subset M$ carrying $\mathscr{F}$ and a finite $R L S$ word $w$ on $\tau$ with $l(w) \leq \Lambda$ so that the semi-infinite $R L S$ word given by the infinite repetition of the word $w$ is convergent and determines the equivalence class of $\mathscr{F}$. Furthermore, the numbers $\lambda$ and $\Lambda$ are related by the inequality

$$
\log \left(\frac{\Lambda}{C_{1}}\right) \leq \lambda^{p\left(\beta^{2}-2 \beta+3\right)} \log 3
$$

where

$$
C_{1}=M_{0} 2^{M_{0}-1}
$$

with

$$
\begin{aligned}
M_{0} & =-6 \chi(M) \\
& =\text { the maximum number of switches of a train track in } M, \\
p= & M_{0} !=\text { the order of the group of permutations on } M_{0} \text { letters, }
\end{aligned}
$$
and

$$
\begin{aligned}
\beta & =-9 \chi(M) \\
& =\text { the maximum number of branches of a train track in } M .
\end{aligned}
$$

Proof. Suppose that $\mathscr{F} \subset M$ is a pseudo-Anosov foliation, which is left invariant by a pseudo-Anosov map $f: M \rightarrow M$ of dilatation $\lambda>1$. By $\S 4$ of [5], there is a train track $\tau \subset M$, say with $J$ branches, so that $f(\tau)$ is carried by $\tau$. We recall that there is a $J$-by- $J$ "incidence matrix" $A$ associated with this carrying defined as follows. Choose a tie neighborhood $N(\tau)$ of $\tau$ in $M$ and a "central tie" in the interior of each maximal rectangle (i.e. a rectangle which is the pre-image 
of a branch of $\tau$ under the natural collapse of $N(\tau)$ to $\tau)$ of $N(\tau)$. The train track $f(\tau)$ may be isotoped to a train track $\tau^{\prime}$ which lies in $N(\tau)$, is transverse to the ties, and whose switches are disjoint from the collection of central ties. The map $f$ induces an identification of the collection of branches of $\tau$ with those of $\tau^{\prime}$, and we define the $(i, j)$ th entry of $A$ to be the number of points of intersection of the $i$ th branch of $\tau^{\prime}$ with the central tie corresponding to the $j$ th branch of $\tau$, for $1 \leq i, j \leq J$.

The incidence matrix $A$ is primitive irreducible (see [2], for instance, for the definition and basic properties of such matrices), the measure on $\tau$ associated to (the projective class of) $\mathscr{F}$ is given by the (unique) positive eigenvector of $A$ with corresponding eigenvalue $\lambda$, and, in fact, $\lambda$ is the spectral radius of $A$; $\mathrm{cf}$. [5].

Suppose that the train track $\tau$ has $I$ switches, and choose a labeling on $\tau$, once and for all. By Lemma 2.1 of [5] and the discussion in $\S 2$ above, there is a finite directed RLS word $w_{0}$ on $\tau$ so that $\tau^{\prime}=\tau\left(w_{0}\right)$. The RLS word $w_{0}$ is uniquely determined given a choice of a linear ordering on the associated pairing arc system (and the conventions in $\S 3$ ), and we may choose the order induced by the labeling on $\tau$.

The one-to-one correspondence between the switches of $\tau$ and those of $\tau^{\prime}$ which is induced by the map $f$ is not necessarily the same as the one induced by the pairing arc system (i.e., by the word $w_{0}$ ). For this reason, we consider the $p$ th power $\Psi=f^{p}$ of $f$, where $p$ is the order of the permutation group on $I$ letters, and define the finite directed RLS word $w$ on $\tau$ arising, as above, from the carrying of $\Psi(\tau)$ by $\tau$. The map $\Psi: M \rightarrow M$ is pseudo-Anosov with dilatation $\lambda^{p}$, and, as explained in [5], the equivalence class of the foliation $\mathscr{F}$ corresponds to the semi-infinite periodic RLS word on $\tau$ which is obtained by infinitely repeating the word $w$. Furthermore, by construction, the bijection between the switches of $\tau$ and the switches of $\Psi(\tau)$ induced by $w$ agrees with the bijection induced by $\Psi$.

We wish to obtain a bound on the word length of $w$ in terms of the dilatation of $\Psi$. Of course, $\Psi(\tau)$ is isotopic to a train track contained in $N(\tau)$, and we let $B$ denote the corresponding incidence matrix, which is primitive irreducible with spectral radius $\lambda^{p}$. By [7], the $\left(b^{2}-2 b+2\right)$ th power of a primitive irreducible $b$-by- $b$ matrix is strictly positive (i.e., each entry of the matrix is strictly positive); it follows that $B^{\prime}=B^{J^{2}-2 J+2}$ is strictly positive.

Now, define the train tracks

$$
\sigma=\Psi^{J^{2}-2 J+2}(\tau) \text { and } \sigma^{\prime}=\Psi(\sigma) .
$$


If we let $w^{n}$ denote the $n$-fold concatenation of the RLS word $n$ with itself for any natural number $n$, then it follows that $\sigma$ is isotopic to $\tau\left(w^{J^{2}-2 J+2}\right)$ and $\sigma^{\prime}$ is isotopic to $\tau\left(w^{J^{2}-2 J+3}\right)$. Indeed, if $w_{1}$ is any RLS word which is equivalent to $w$, then it is also true that $\sigma$ is isotopic to $\tau\left(w_{1}^{J^{2}-2 J+2}\right)$ and $\sigma^{\prime}$ is isotopic to $\tau\left(w_{1}^{J^{2}-2 J+3}\right)$.

It is well-known (see [2] for instance) that the spectral radius of a primitive irreducible nonnegative matrix is bounded below by its least column sum. Since $B^{\prime}$ is strictly positive, it follows easily that the spectral radius of $B^{\prime}$ is at least $J$. We wish to use this estimate to find a lower bound on the least column sum of $B^{\prime \prime}=B B^{\prime}=B^{J^{2}-2 J+3}$ in terms of the word length $l=l(w)$ of $w$. By definition, for each $j=1, \ldots, J$, the $j$ th column sum of $B^{\prime \prime}$ is equal to the number of points of intersection of the train track $\sigma^{\prime}$ with the central tie of $N(\tau)$ corresponding to the $j$ th branch of $\tau$.

By Proposition 4 above, we may re-order the pairing arcs corresponding to the unzipping of $\tau$ to $\sigma$ associated to $w$ to produce a finite directed RLS word $w^{\prime}$ which is equivalent to $w$ so that the arc length $l_{1}^{\prime}$ of the first pairing arc $\Gamma$ corresponding to $w^{\prime}$ is at least $l / C_{1}$, where the constant $C_{1}$ is given in the proposition and depends only on the Euler characteristic of $M$. We may regard $w^{\prime}$ as a finite directed RLS word on $\sigma$ itself in the natural way and observe that $\sigma^{\prime}=\sigma\left(w^{\prime}\right)$.

Consider the sequence

$$
\sigma=\sigma_{0}, \sigma_{1}, \ldots, \sigma_{K}=\sigma^{\prime}
$$

of train tracks associated to the RLS word $w^{\prime}$, where each $\tau_{k+1}$ arises from $\tau_{k}$ by an elementary move, for $k=0, \ldots, K-1$.

We claim that if $t$ is a central tie of $N(\tau)$, then

$$
\#\left\{t \cap \sigma_{k+1}\right\} \geq \#\left\{t \cap \sigma_{k}\right\}+1
$$

for each $k=0, \ldots,\left[\log l_{1}^{\prime} / \log 3\right]-2$. To see this, notice that by Proposition 5 above, the unzipping along $\Gamma$ involves at least $\left[\log l_{1}^{\prime} / \log 3\right]$ elementary moves. Insofar as each branch of $\sigma$ meets each central tie of $N(\tau)$ (since $B^{\prime}$ is strictly positive), each of these elementary moves, except perhaps the last one, involves a segment of $\Gamma$ which meets each central tie of $N(\tau)$ at least once, and the claim is proved.

Finally, if $t$ is a central tie of $N(\tau)$, then, since each pairing arc is transverse to the ties of $N(\tau)$,

$$
\#\left\{t \cap \sigma_{k+1}\right\} \geq \#\left\{t \cap \sigma_{k}\right\}
$$


for each $k=0, \ldots, K-1$; see Figure 7. It follows directly that each column sum of $B^{\prime \prime}$ is at least $\left[\log l_{1}^{\prime} / \log 3\right]-1+J$. The estimate

$$
\lambda^{p\left(J^{2}-2 J+3\right)} \geq\left[\frac{\log l_{1}^{\prime}}{\log 3}\right]+J-1
$$

follows (since the spectral radius is bounded below by the least column sum), and the proof of the desired inequality is therefore complete.
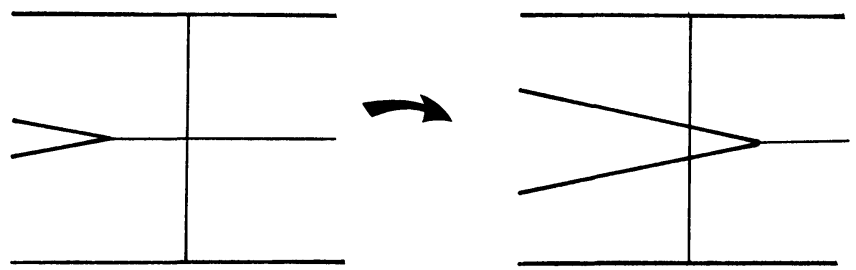

\section{FIGURE 7}

6. Enumerating conjugacy classes of pseudo-Anosov foliations. By Theorem 6 above, for any real number $R$, there is an integer $\Lambda$ so that every pseudo-Anosov foliation in the surface $M$ which is preserved by some pseudo-Anosov map of dilatation at most $R$ is determined by the infinite repetition of a directed finite RLS word $w$ on some train track in $M$, where the word length of $w$ is at most $\Lambda$; furthermore, $\Lambda$ is bounded in terms of $R$ by the inequality

$$
R^{p\left(\beta^{2}-2 \beta+3\right)} \geq \frac{\log \Lambda / C_{1}}{\log 3},
$$

where $p, \beta$, and $C_{1}$ are numbers which depend only on the Euler characteristic of the surface $M$.

Let us therefore consider a finite collection $\mathscr{S}$ of (unlabeled) train tracks in $M$, one representative train track from each conjugacy class of train tracks in $M$ each of whose complementary regions is simply connected, and choose a labeling on each $\tau \in \mathscr{S}$. For each $\tau \in \mathscr{S}$, consider the collection $W_{R}(\tau)$ of all (equivalence classes of) finite directed RLS words of word length at most $\Lambda$ on $\tau$. It follows that if $\mathscr{F}$ is a pseudo-Anosov foliation of $M$ which is preserved by a pseudoAnosov map of $M$ of dilatation at most $R$, then $\mathscr{F}$ arises from the infinite repetition of some word $w \in W_{R}(\tau)$ for some $\tau \in \mathscr{S}$.

This gives an effective upper bound (in terms of the topology of the surface $M$ ) on the number of distinct conjugacy classes of pseudoAnosov foliations in $M$ whose corresponding dilatations have some a priori upper bound. 


\section{REFERENCES}

[1] A. Fathi, F. Laudenbach and V. Poenaru, Travaux de Thurston sur les Surfaces, Astérisque, 66-67 (1979).

[2] F. R. Gantmacher, The Theory of Matrices, Chelsea (1959).

[3] R. C. Penner and J. Harer, Combinatorics of train tracks, pre-print.

[4] A. Papadopoulos, Réseaux ferroviàires, difféomorphismes pseudo-Anosov et automorphismes symplectiques de l'homologie, Publ. Math. d'Orsay, $\mathrm{n}^{\circ}$ 83-03 (1983).

[5] A. Papadopoulos and R. C. Penner, A characterization of pseudo-Anosov foliations, Pacific J. Math., 130 (1987), 359-377.

[6] W. P. Thurston, The Geometry and Topology of 3-Manifolds, Princeton Univ. Lecture Notes, (1978).

[7] H. Wielandt, Unzerlegbare nicht negative Matrizen, Math. Zeitschrift, 52 (1950), 642-648.

Received March 10, 1987 and in revised form October 31, 1988. Partially supported by CNRS (France) and the National Science Foundation (USA).

C.N.R.S.

UNiversité Louis Pasteur

7 RUE RENÉ DESCARTES

67084, Strasbourg, France

AND

UNIVERSITY OF SOUTHERN CALIFORNIA

Los ANGeles, CA 90089-1113 



\section{PACIFIC JOURNAL OF MATHEMATICS EDITORS}

\author{
V. S. VARADARAJAN \\ (Managing Editor) \\ University of California \\ Los Angeles, CA 90024-1555-05 \\ Herbert Clemens \\ University of Utah \\ Salt Lake City, UT 84112 \\ ThOMAS ENRIGHT \\ University of California, San Diego \\ La Jolla, CA 92093
}

\section{R. FINN}

Stanford University

Stanford, CA 94305

HeRmann FlaschKa

University of Arizona

Tucson, AZ 85721

VAUGHAN F. R. JONES

University of California

Berkeley, CA 94720

Steven KerckhofF

Stanford University

Stanford, CA 94305

\author{
RobION KIRBY \\ University of California \\ Berkeley, CA 94720 \\ C. C. Moore \\ University of California \\ Berkeley, CA 94720 \\ HAROLD STARK \\ University of California, San Diego \\ La Jolla, CA 92093
}

\section{ASSOCIATE EDITORS}
R. ARENS
E. F. BECKenBach
B. H. NeumanN
F. WOLF
K. YoshidA (1906-1982)
(1904-1989)

\section{SUPPORTING INSTITUTIONS}

UNIVERSITY OF ARIZONA

UNIVERSITY OF BRITISH COLUMBIA

CALIFORNIA INSTITUTE OF TECHNOLOGY

UNIVERSITY OF CALIFORNIA

MONTANA STATE UNIVERSITY

UNIVERSITY OF NEVADA, RENO

NEW MEXICO STATE UNIVERSITY

OREGON STATE UNIVERSITY
UNIVERSITY OF OREGON

UNIVERSITY OF SOUTHERN CALIFORNIA

STANFORD UNIVERSITY

UNIVERSITY OF HAWAII

UNIVERSITY OF TOKYO

UNIVERSITY OF UTAH

WASHINGTON STATE UNIVERSITY

UNIVERSITY OF WASHINGTON 


\section{Pacific Journal of Mathematics}

Vol. 142, No. $1 \quad$ January, 1990

Marco Andreatta, Mauro Beltrametti and Andrew Sommese, Generic properties of the adjuction mapping for singular surfaces and applications

Chen-Lian Chuang and Pjek-Hwee Lee, On regular subdirect products of

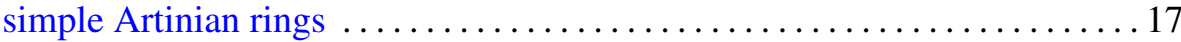

Fernando Giménez and Vicente Miquel Molina, Volume estimates for real hypersurfaces of a Kaehler manifold with strictly positive holomorphic sectional and antiholomorphic Ricci curvatures $\ldots \ldots \ldots \ldots \ldots \ldots 23$

Richard J. Griego and Andrzej Korzeniowski, Asymptotics for certain Wiener integrals associated with higher order differential operators

Abdeslam Mesnaoui, Unitary bordism of classifying spaces of quaternion

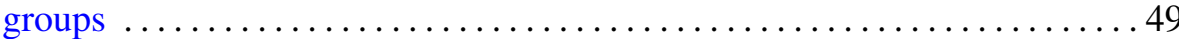

Abdeslam Mesnaoui, Unitary cobordism of classifying spaces of quaternion

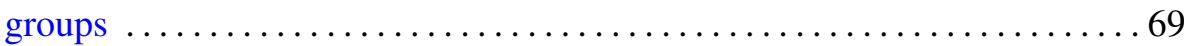

Jesper M. Møller, On equivariant function spaces $\ldots \ldots \ldots \ldots \ldots \ldots \ldots \ldots$

Bassam Nassrallah, A $q$-analogue of Appell's $F_{1}$ function, its integral representation and transformations

Peter A Ohring, Solvability of invariant differential operators on metabelian

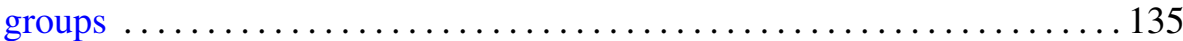

Athanase Papadopoulos and R. C. Penner, Enumerating pseudo-Anosov foliations

Ti-Jun Xiao and Liang Jin, On complete second order linear differential

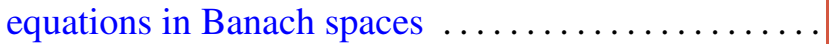

Carl Widland and Robert F. Lax, Weierstrass points on Gorenstein curves 